\title{
Herramienta para predecir la gravedad y secuelas de la COVID-19 en sanitarios del entorno de hospitales. El "COVID-19 Occupational Vulnerability Index"
}

\author{
Association Between the "COVID-19 Occupational \\ Vulnerability Index" and COVID-19 Severity and \\ Sequelae Among Hospital Employees
}

\author{
M $^{\mathrm{a}}$ Teófila Vicente-Herrero ${ }^{1}$ (1) 0000-0002-0796-9194 \\ Alejandro Fernandez-Montero² (1) 0000-0002-8741-7401 \\ 'Servicio Medicina del Trabajo-Correos Valencia, España. \\ ${ }^{2}$ Área de Medicina del Trabajo, Clínica Universidad de Navarra, Pamplona, España.
}

\section{Resumen}

Este trabajo es un comentario del artículo: Navarro-Font X, Kales SN, Vicente-Herrero MT, Rueda-Garrido JC, Del Campo MT, Reinoso-Barbero L, Fernandez-Montero A. Association Between the "COVID-19 Occupational Vulnerability Index" and COVID-19 Severity and Sequelae Among Hospital Employees. J Occup Environ Med. 2021;63(10):895-900. doi: 10.1097/ JOM.0000000000002253.

\section{Abstract}

This text is a commentary on the article: Navarro-Font X, Kales SN, Vicente-Herrero MT, Rueda-Garrido JC, Del Campo MT, Reinoso-Barbero L, Fernandez-Montero A. Association Between the "COVID-19 Occupational Vulnerability Index" and COVID-19 Severity and Sequelae Among Hospital Employees. J Occup Environ Med. 2021;63(10):895-900. doi: 10.1097/ JOM.0000000000002253.

Fechas - Dates

Recibido: 2021.10 .10 Aceptado: 2020.10.10

Publicado: 2021.10.15
Sección coordinada por

Consol Serra (consol.serra@upf.edu) Ma del Mar Seguí (mm.Segui@ua.es) 


\section{Resumen del artículo comentado}

Fundamentos: Además de los factores personales y relacionados con la salud, los trabajadores sanitarios tienen un mayor riesgo debido a su trabajo. Evaluamos la asociación de la puntuación del Índice de Vulnerabilidad Laboral con el riesgo de sufrir una COVID-19 grave y sus secuelas.

Métodos: Estudio observacional retrospectivo realizado en trabajadores sanitarios. Entre 119 empleados infectados, se calculó el Índice de Vulnerabilidad Laboral COVID-19 (compuesto por 29 ítems relativos a la salud personal, las condiciones de trabajo y la capacidad de cumplir con las medidas preventivas) y se correlacionó con la gravedad/secuelas de la COVID-19.

Resultados: Los trabajadores con puntuaciones más altas (de seis a siete puntos) tuvieron un riesgo significativamente mayor de desarrollar enfermedad grave (OR =9,73; IC 95\%, 1,53 a 35,56) y secuelas clínicas $(O R=5,22$; IC 95\%, 1,80 a 15,16) que aquellos con puntuaciones más bajas ( 0 a 3).

Conclusiones: El "COVID-19 Occupational Vulnerability Index" puede predecir el riesgo de enfermedad grave por COVID-19 y las secuelas clínicas entre los trabajadores sanitarios.

\section{Comentario}

En los últimos meses se ha publicado numerosos artículos en las principales revistas médicas sobre el la infección por el coronavirus y el síndrome respiratorio agudo severo 2 (SARS-CoV-2). La afección resultante, la enfermedad por coronavirus 2019 (COVID-19), ha tenido un efecto social sólo comparable al de la epidemia de gripe española de 1918. Por lo que de forma progresiva se ha ido conociendo más sobre qué individuos y grupos experimentan las complicaciones más graves. Se sabe que variables personales como la edad y las comorbilidades influyen en la vulnerabilidad de las personas al COVID-19 y se asocian con la gravedad de la infección y su letalidad ${ }^{(1,2)}$. Estos factores de riesgo han sido incluidos para la tipificación de trabajador vulnerable en las empresas por parte de Ministerio de Sanidad con las revisiones pertinentes según la evolución de la pandemia(3).

Además, las condiciones de trabajo del personal sanitario y su capacidad para cumplir con otras medidas de prevención adecuadas, han demostrado ser importantes para disminuir las tasas de infección entre el personal hospitalario(4).

En Salud Laboral, ha sido destacable la labor realizada por los profesionales de los Servicios de Prevención, y de forma especial la de los sanitarios en cuanto a valorar la compatibilidad del desempeño laboral, minimizando el riesgo de contagio y las consecuencias en personas o colectivos especialmente vulnerables. Por ello, se han desarrollado propuestas de valoración de vulnerabilidad laboral frente a COVID-19 para reducir al máximo esta probabilidad de ser afectado por el virus con riesgo superior al normal y con consecuencias más graves, ya sea como 
resultado de la susceptibilidad mayor a los efectos del virus, o por un nivel de exposición superior a la media(5).

La Organización Mundial de la Salud nos anima a pensar de manera innovadora y la promoción de la salud y prevención de riesgos puede liderar esta actividad dentro del ámbito de salud pública, obteniendo información precisa y oportuna para prepararse y reducir el riesgo en las personas afectadas, sus familias y su comunidad.

Dada la complejidad de este tema y los distintos aspectos que se han de tener en cuenta para calificar a un trabajador como vulnerable frente a esta infección pandémica, se ha considerado de utilidad incluir en la valoración aspectos personales de la persona que trabaja, enfermedades previas y su grado de control, aspectos laborales y las opciones de gestión preventiva de las empresas.

El resultado de esta combinación de factores ha permitido cuantificar la vulnerabilidad individual y orientar las actuaciones preventivas posteriores en el mundo del trabajo en forma de un índice: el de vulnerabilidad laboral(6). Se trata de un índice elaborado por los autores y en el que hay que considerar como limitación no tener una validación formal, si bien los resultados obtenidos hasta el momento con su utilización son muy indicativos y permiten usar la herramienta con cautela y rigor.

Este índice de vulnerabilidad se ha aplicado de forma concreta en un colectivo de sanitarios de ámbito hospitalario en los que se evaluó la asociación de la puntuación del Índice "COVID-19 Occupational Vulnerability Index" con el riesgo de sufrir una COVID-19 grave y sus secuelas. Para ello se realizó un estudio observacional retrospectivo en 119 empleados infectados, se calculó el Índice de Vulnerabilidad Laboral COVID-19 (compuesto por los 29 ítems) y se correlacionó con la gravedad/secuelas de COVID-19.

Los resultados muestran que los trabajadores con puntuaciones más altas (6 a 7) tenían un riesgo significativamente mayor de desarrollar una enfermedad grave $(\mathrm{OR}=9,73$; IC del 95\%, 1,53 a 35,5). y secuelas clínicas (OR=5,22; IC del 95\%: 1,80 a 15,16$)$ frente a aquellos con puntuaciones más bajas (0 a 3). Esto permite concluir que, si bien deben realizarse futuros estudios prospectivos con muestras de población más amplias y multicéntricas, el "COVID-19 Occupational Vulnerability Index" puede ser una herramienta útil para discriminar el riesgo de enfermedad grave por COVID-19 y sus secuelas, entre los trabajadores de un entorno sanitario.

\section{Referencias}

1. Zhou F, Yu T, Du R, et al. Clinical course and risk factors for mortality of adult inpatients with COVID-19 in Wuhan, China: a retrospective cohort study. Lancet. 2020;395:1054-1062.

2. Starke KR, Petereit-Haack G, Schubert $M$, et al. The age-related risk of severe outcomes due to covid-19 infection: a rapid review, meta-analysis, and meta-regression. Int J Environ Res Public Health. 2020;17:1-24. 
3. Ministerio de Sanidad. Procedimiento de actuación para los servicios de prevención de riesgos laborales frente a la exposición al SARS-CoV-2. Julio 2021 [actualizado 10 Oct 2021; citado 21 Oct 2021]. Disponible en: https://www.mscbs.gob. es/profesionales/saludPublica/ccayes/alertasActual/nCov/documentos/Proteccion_Trabajadores_SARS-CoV-2.pdf

4. Chou R, Dana T, Buckley DI, Selph S, Fu R, Totten AM. Epidemiology of and risk factors for coronavirus infection in health care workers: a living rapid review. Ann Intern Med. 2020;173:120-136.

5. Vicente-Herrero T, de la Torre VRI, del Campo Balsa T, Barbero LR, Montero AF, Garrido JCR. Proposed protocol for risk assessment and stratification. Occup Dis Environ Med. 2020;08:99-110. doi: 10.4236/odem.2020.83008.

6. Vicente-Herrero MT, Ramírez Iñiguez de la Torre MV, del Campo Balsa MT, Reinoso Barbero L, Rueda Garrido JC, Santamaría Navarro C. Proposed occupational vulnerability index COVID-19. Occup Dis Environ Med. 2020;08:175-187. doi: 10.4236/odem.2020.84014 